\title{
Internal fixation of proximal humeral fractures with locking proximal humeral plate (LPHP) in elderly patients with osteoporosis
}

\author{
Ramchander Siwach $\cdot$ Roop Singh • \\ Rajesh Kumar Rohilla • Virender Singh Kadian • \\ Sukhbir Singh Sangwan $\cdot$ Manjeet Dhanda
}

Received: 16 December 2007/ Accepted: 12 May 2008/Published online: 16 July 2008

(C) Springer-Verlag 2008

\begin{abstract}
Background Different operative techniques used for treating displaced proximal humeral fractures could result in malunion, non-union, osteonecrosis of humeral head, loosening of screw and loss of reduction particularly in comminuted and osteoporotic fractures. Locking compression plate (LPHP) has been proposed for open reduction and internal fixation of these fractures and is associated with less complication rate.

Materials and methods We prospectively assessed the functional outcome and the complications after an average follow-up of 24.9 months in 25 patients of proximal humeral fractures with osteoporosis. Mean age was 62 years. Using AO classification, $48 \%$ were type A and $52 \%$ type B.

Results Mean constant score was 80 points. According to constant score, $28 \%$ had excellent outcome, $64 \%$ had good functional outcome, and $8 \%$ had moderate outcome. When the results were related to grades of osteoporosis, grade IV osteoporotic fractures had highest average Constant-Murley score ( 83 points, range $78-88$ points), followed by grade III osteoporotic fractures (80 points, range 71-92 points), followed by grade II osteoporotic fractures (78 points, range $66-88$ points). Varus malalignment and subacromial impingement were observed in $8 \%$ patients. Loosening of implant and loss of reduction were observed
\end{abstract}

R. Siwach - R. Singh - R. K. Rohilla - V. S. Kadian ·

S. S. Sangwan $\cdot$ M. Dhanda

Department of Orthopaedic Surgery,

Paraplegia and Rehabilitation, Pt. B.D. Sharma PGIMS,

Rohtak, 124001, Haryana, India

R. Siwach $(\bowtie)$

9-J/52, Medical Enclave PGIMS, Rohtak 124001, Haryana, India

e-mail: drroopsingh@ rediffmail.com in $4 \%$ patients. Superficial infection was observed in $4 \%$ patients.

Conclusions Locking compression plate (LPHP) is an advantageous implant in proximal humeral fractures due to angular stability, particularly in comminuted fractures and in osteoporotic bones in elderly patients, thus allowing early mobilization.

Keywords Locking compression plate · Fractures · Proximal · Humerus · Internal fixation · Osteoporosis

\section{Introduction}

Proximal humeral fractures are the third most common fracture in the elderly patients [1]. The incidence of proximal humeral fractures is increasing, probably due to the ageing society and the associated increase in the incidence of osteoporosis [1, 2]. Conservative treatment of displaced proximal humeral fractures may result in unacceptable deformity and stiffness of the shoulder [3, 4]. Different operative techniques used for treating displaced proximal humeral fractures can result in malunion, nonunion and osteonecrosis of humeral head [5-7]. Comminuted fractures and older patients presenting with weakened bone from osteoporosis present additional challenge to treatment [2]. As proximal fragment is too small to accommodate minimum of three screws, loosening of screws and loss of reduction may occur with conventional implants $[6,8]$. Poor rotational and angular stability can lead to a partial loss of reduction into varus or retro flexion, resulting in an unsatisfactory functional outcome [3].

For full functional recovery of shoulder anatomical reduction, stable fixation and early mobilization are required. Recently, locking proximal humeral plate 
(LPHP), providing greater angular stability, has been proposed for operative fracture treatment [9]. Its theoretical advantage is better anchorage of screws in osteoporotic bone. Because of the good fixation, there is potential of enhanced stability that could allow early mobilization. Additionally, they can be inserted using a minimally invasive technique without additional trauma to the soft tissues [10].

The objective of this study was to evaluate the clinical outcome and complications of LPHP in managing displaced proximal humeral fractures with osteoporosis in elderly patients.

\section{Materials and methods}

This was a prospective study performed at Department of Orthopaedics, PGIMS, Rohtak between April 2003 and May 2007. A total of 25 patients (12 males and 13 females) with displaced proximal humeral fractures were operated using LPHP.

The inclusion criteria into the study were

1. Closed displaced two- and three-part proximal humeral fractures in adults with osteoporosis.

2. Failed conservative treatment (unsatisfactory position).

3. Patients without neurological deficit.

4. Grade I-IV osteoporosis as per Singh's index [11].

Open fractures, pathological fractures, and four-part fractures were excluded from study.

All proximal humeral fractures met the indications for the operative treatment outlined by Neer [4] i.e. an angulation of articular surface of more than $45^{\circ}$, a displacement between the major fracture segments more than $1 \mathrm{~cm}$, or a fracture with valgus impaction [15]. Two-part fractures involving only the greater or lesser tuberosity were not included in this study, since they were managed by screw fixation. Mean age was 62 years (range 54-69 years). Mean follow-up time was 24.9 months (range 1528 months). Using plain radiographs, all fractures were classified according to AO classification and Neer classification [12]. Table 1 shows classification of fractures in the series. Antero-posterior radiographs of pelvis including both hips were assessed for grading osteoporosis. Using $\mathrm{AO}$ classification, five fractures were type $\mathrm{A} 2$, seven were $\mathrm{A} 3$, four were B1, seven were B2, and two fractures were B3. According to Neer classification, 12 fractures were 2part and 13 fractures were 3-part. The causes of injury were falls (17), motor vehicle accidents (5) and assaults (3).

All patients received a prophylactic dose of $1 \mathrm{gm}$ cefoperazone + sulbactum intravenously preoperatively. The operation was done in supine position with small sand bag under shoulder, under general anaesthesia. Fracture was
Table 1 Classification according to Neer and AO/ASIF of the proximal humeral fractures in a series of 25 patients treated with locking proximal humeral plate

\begin{tabular}{|c|c|c|c|c|c|}
\hline Neer & $n$ & $\mathrm{AO}$ & & $n$ & Subtotal \\
\hline \multirow[t]{4}{*}{ 2-Part } & 12 & Type-A & 2.2 & 5 & 12 \\
\hline & & & 3.1 & 2 & \\
\hline & & & 3.2 & 4 & \\
\hline & & & 3.3 & 1 & \\
\hline \multirow[t]{5}{*}{ 3-Part } & 13 & Type-B & 1.3 & 4 & 13 \\
\hline & & & 2.1 & 3 & \\
\hline & & & 2.3 & 4 & \\
\hline & & & 3.1 & 1 & \\
\hline & & & 3.2 & 1 & \\
\hline Total & 25 & & & & 25 \\
\hline
\end{tabular}

exposed through delto-pectoral approach. Fracture fragments were reduced without stripping periosteum to maximum possible achievable anatomical position and reduction was held with Kirschner wires. Reduction was checked under image intensifier. Definitive fixation with locking proximal humeral plate was done with plate positioned lateral to bicipital groove sparing tendon of long head of biceps. The plate was placed at least $1 \mathrm{~cm}$ distal to the upper end of greater tubercle. Plate was fixed with screw at longitudinal dynamic hole. After achieving near anatomical reduction, multidirectional screws were used to fix proximal fragments. Meticulous repairs of the rotator cuff, capsule and subscapularis muscle tears/avulsions were carried out, if found pre-operatively. Lesser tuberosity was fixed with a separate screw/wire if found avulsed. Range of motion of shoulder was checked on the table for impingement. Wound was closed under negative suction, which was removed after 48 hours. The patient were followed up at 15 days, then monthly for 6 months, and then at 12 months for final evaluation. Standard anteroposterior and axillary radiographs were obtained and evaluated for bony healing, non-union, malunion, loosening of implant, loss of reduction and avascular necrosis of head of humerus. Comparing the immediate postoperative radiographs and those taken at the time of the final assessment assessed loss of reduction. Assessment and analysis of any complications including axillary nerve injury and impingement due to plate was done. Functional outcome was assessed according to Constant-Murley score [13]. The Constant-Murley score was graded as poor (0-55 points), moderate (56-70), good (71-85), or excellent (86-100).

Ethical considerations

Informed consent was taken from the patients prior to operation and for the inclusion to the study. The study was 
performed according to the Declaration of Helsinki, and the Institutional Ethical Board approved it.

\section{Results}

In this study, falls accounted for majority of fractures i.e. in $68 \%$ patients, motor vehicle accidents in $20 \%$ patients and assaults accounted for $12 \%$ patients. Tables 2 and 3 show functional outcome presented as Constant-Murley score at 3, 6 and 12 months follow-up according to fracture type and grades of osteoporosis, respectively. Mean ConstantMurley score was 80 points at one-year follow-up. According to constant score, $28 \%$ had excellent outcome, $64 \%$ had good functional outcome and $8 \%$ had moderate outcome. All fractures united with an average union time of 18 (16-23) weeks (Figs. 1, 2). When the results were related to fracture classification, two-part fractures had the higher average Constant-Murley score (83 points, range 71-92 points) as compared to three-part fractures (78 points, range $66-88$ points). When the results were related to grades of osteoporosis, grade IV osteoporotic fractures had highest average Constant-Murley score (83 points, range 78-88 points), followed by grade III osteoporotic fractures (80 points, range $71-92$ points), followed by grade II osteoporotic fractures (78 points, range 66-88 points). In two patients, fracture had varus malalignment. In two patients, there was subacromial impingement. No patient had axillary nerve paresis. No deep wound infection, vascular injuries or osteonecrosis of head were noted. Loosening of implant was observed in one patient. However, fracture united without the need of refixation.

Table 2 Functional outcome in different fracture types, presented as mean and range of the Constant score at 3,6 and 12 months follow-up

\begin{tabular}{llll}
\hline Follow-up & All $(n=25)$ & \multicolumn{2}{l}{ Constant score according to fracture type } \\
\cline { 3 - 4 } & & 2-Part $(n=12)$ & 3-Part $(n=13)$ \\
\hline 3 Months & $69(60-80)$ & $72(60-80)$ & $66(61-78)$ \\
6 Months & $75(60-86)$ & $78(67-86)$ & $73(60-84)$ \\
12 Months & $80(66-92)$ & $83(71-92)$ & $78(66-88)$ \\
\hline
\end{tabular}

Table 3 Functional outcome in different grades of osteoporosis presented as mean and range of the Constant score at 3, 6 and 12 months follow-up

\begin{tabular}{llll}
\hline Follow-up & \multicolumn{3}{l}{ Constant score according to grades of osteoporosis } \\
\cline { 2 - 4 } & II $(n=8)$ & III $(n=9)$ & IV $(n=8)$ \\
\hline 3 Months & $65(55-76)$ & $68(60-80)$ & $73(66-80)$ \\
6 Months & $72(60-82)$ & $75(67-86)$ & $78(68-86)$ \\
12 Months & $78(66-88)$ & $80(71-92)$ & $83(78-88)$ \\
\hline
\end{tabular}

Superficial infection occurred in one patient, it resolved with antibiotic coverage.

\section{Discussion}

Displaced proximal humeral fractures in elderly patients pose a challenge to treatment when associated with osteoporosis and comminution. Osteoporosis predispose to low energy fractures which often have a complex pattern [14]. Poor bone quality makes screw purchase and fixation less secure $[6,8]$. The decreased healing capacity in osteoporosis is reflected in a dramatic increase in the rate of failure of implant fixation [14, 15].

In present study, LPHP has shown encouraging results in displaced proximal humeral fractures in osteoporotic bones. Sound union was achieved in all patients. Secondary loss of reduction occurred in $4 \%$ patients after screw loosening in proximal fragment. Secondary varus deformity (head-shaft axis angle $<120^{\circ}$ ) and retroversion of humeral head occurred in $14 \%$ patients in conventional plate osteosynthesis. Bone cement had been used to improve the holding power of screws in osteoporotic bones. Implant failure with screw loosening and secondary displacement of fracture fragments necessitated refixation of fracture in $4 \%$ patients [3]. No revision surgery was performed in our study due to implant failure. LPHP was associated with significant lower risk of screw loosening and secondary loss of reduction as compared to conventional plates in the present series. LPHP offers the advantage of locking head screws, which enter the humeral head at various angles in order to maximise purchase [14]. Fracture in a poor position is associated with poor functional results $[3,5]$. Malunion was mainly a hardware related problem. Insufficient fixation of the screws may cause partial loss of reduction with secondary displacement of the humeral head into varus position leading to unsatisfactory result. Whereas, a higher rate (12\%) of varus malunion was observed in conventional plate osteosynthesis [3]. We did not have any secondary varus deformity. However, fracture was fixed in varus primarily in $8 \%$ patients in our series and both these patients had moderate outcome. Primary malunion can be prevented if fracture is fixed in near anatomical position at the time of fixation. We feel that near anatomical reduction must be achieved before applying multidirectional screws, as plate does not help in reduction of proximal fragments. Rather it fixes the proximal fragments wherever they are. With varus malalignment, the plate must not be positioned too far cranially, otherwise there could be subacromial impingement which occurred in our two patients with varus malnion. Wanner et al. [16] treated displaced proximal humerus fractures with open reduction and internal fixation with two one-third tubular plates on the anterior and lateral aspects of the proximal humerus. High 
Fig. 1 a AO 11A 3 fracture in a patient with Singh's grade II osteoporosis. b Anteroposterior radiograph of same patient 12 months postoperatively. c, d Postoperative functional photographs of same patient
Fig. 2 a AO 11B 1 fracture in a patient with Singh's grade III osteoporosis. b Anteroposterior radiograph of same patient 12 months postoperatively. c, d Postoperative functional photographs of same patient
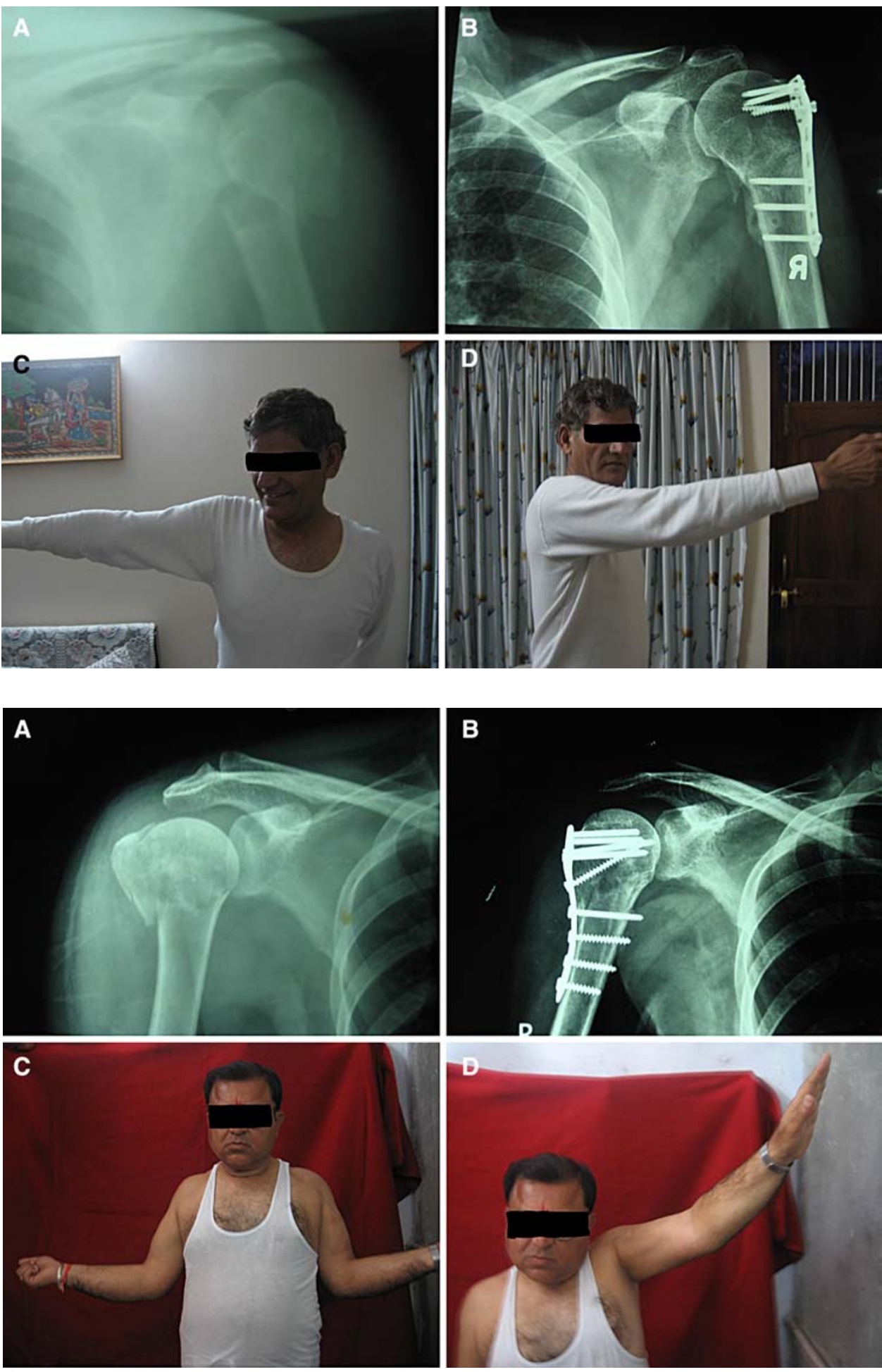

stability, thus achieved, allowed early mobilization of the shoulder. Fixed angled devices, such as the angled blade plate, are very useful as they resist angular deformation and torsion [14, 17]. However, Meier et al. [18] did not recommend internal fixation with angled blade plate in unstable proximal humerus fractures due to high rate of complications (33\%) including protrusion of blade into glenohumaral articulation (22\%). Several authors showed satisfactory results with implants providing an angular stability $[9,10$, $19,20]$. Superficial infection rate of $4 \%$ in our series is comparable to $5 \%$ in series by Kaukakis et al. [20]. Avascular necrosis of humerus has been reported to be $4-5 \%$ in other series $[9,20]$. Only AO/ASIF type-C fracture or Neer's 4-part fracture had this complication. We did not include 
AO/ASIF type-C fracture or Neer's 4-part fracture in our series. This may be the one of the reasons for non-occurrence of avascular necrosis in our series. Our results are comparable with other series using implants providing an angular stability with respect to union, subacromial impingement, secondary loss of reduction and varus malunion $[9,20]$. This suggests that LPHP is associated with satisfactory results in both osteoporotic and non-osteoporotic fractures of proximal humerus. Although small no of patients in our series is an limitation, higher rate of secondary loss of reduction (12.5\%) was observed in fractures with severe osteoporosis (Singh index grade II) as compared to fractures with mild osteoporosis $(0 \%)$ (Singh index grade IV). This suggests that there is need for further improvement in management of osteoporotic proximal humeral fractures.

The goal of surgical therapy is to obtain fracture reduction and stable fixation to enable immediate functional after treatment without the need for postoperative immobilization [3]. The LPHP demonstrated superior biomechanical characteristics compared with the proximal humeral nail [21]. Additional holes in the plate allow tension band fixation of the rotator cuff $[9,14]$. Stable construct allows early mobilization and satisfactory functional outcome. Use of LCP is recommended in elderly patients with osteoporotic bone [9]. We are also of this view as elderly patients could attain an activity level that was sufficient to satisfy their needs regarding independent daily living. But, as expected, the mean Constant-Murley score declined with increasing age. It is because after achieving a satisfactory functional result with a good range of motion, elderly patients usually discontinue exercise at home and often lose range of motion. Author of this series has experience of open reduction and internal fixation on proximal humeral fractures and fracture dislocations using T-plate and bent semitubular plate (employed as a blade plate) in 1990 [22]. Previous study had poor outcome in elderly patients. With the experience of both techniques, we have found locking proximal humeral plate an advantageous implant in communited 2-part fractures, 3-part fractures with osteoporosis in elderly patients. Fixed angular stability and meticulous rotator cuff repair leads to early mobilization and satisfactory functional outcome.

Conflict of interest statement No financial grants have been received for the study. No other people or organization is associated with the study to influence the work.

\section{References}

1. Palvanen M, Kannus P, Niemi S, Parkkari J (2006) Update in the epidemiology of proximal humeral fractures. Clin Orthop 442:87-92
2. Rees J, Hicks J, Ribbans W (1998) Assessment and management of three- and four-part proximal humeral fractures. Clin Orthop 353:18-29

3. Hessmann M, Baumgaertel F, Gehling H, Klingelhoeffer I, Goetzen L (1999) Plate fixation of proximal humeral fractures with indirect reduction: Surgical technique and results utilizing three shoulder scores. Injury 30:453-462

4. Neer CS (1970) Displaced proximal humeral fractures-II. Treatment of three-part and four-part displacement. J Bone Joint Surg 52A:1090-1103

5. Gerber C, Werner CM, Vienne P (2004) Internal fixation of complex fractures of the proximal humerus. J Bone Joint Surg 86B:848-55

6. Szyszkowitz R, Seggl W, Schleifer P, Cundy PJ (1993) Proximal humeral fractures. Management techniques and expected results. Clin Orthop 292:13-25

7. Wijgman AJ, Roolker W, Patt TW, Raaymakers EL, Marti RK (2002) Open reduction and internal fixation of three and four-part fractures of the proximal part of humerus. J Bone Joint Surg 84A:1919-25

8. Cofield RH (1988) Comminuted fractures of the proximal humerus. Clin Orthop 230:49-57

9. Bjorkenheim JM, Pajarinen J, Savolainen V (2004) Internal fixation of proximal humeral fractures with a locking compression plate. A retrospective evaluation fo 72 patients followed for a minimum of 1 year. Acta Orthop Scand 75:741-745

10. Helmy N, Hintermann B (2006) New trends in the treatment of proximal humerus fractures. Clin Orthop 442:100-8

11. Singh M, Nagrath AR, Maini PS (1970) Changes in the trabecular pattern of the upper end of the femur as an index of osteoporosis. J Bone Joint Surg 52A:457-467

12. Neer CSII (1970) Displaced proximal humeral fractures. Part I. Classification and evaluation. J Bone Joint Surg 52A:1077-89

13. Constant CR, Murley AH (1987) A clinical method of functional assessment of the shoulder. Clin Orthop 214:160-4

14. Hertel R (2005) Fractures of the proximal humerus in osteoporotic bone. Osteoporos Int 16(Suppl 2):S65-S72

15. Giannoudis PV, Schneider E (2006) Principles of fixation of osteoporotic fractures. J Bone Joint Surg 88B:1272-8

16. Wanner GA, Wanner-Schmid E, Romero J, Hersche O, Von Smekal A, Trentz O, Ertel W (2003) Internal fixation of displaced proximal humeral fractures with two-one-third tubular plates. J Trauma 54(3):536-544

17. Hintermann B, Trouillier HH, Schafer D (2000) Rigid internal fixation of fractures of the proximal humerus in older patients. $\mathbf{J}$ Bone Joint Surg 82B:1107-1112

18. Meier RA, Messmer P, Regazzoni P, Rothfischer W, Gross T (2006) Unexpected high complication rate following internal fixation of unstable proximal humerus fractures with an angled blade plate. J Orthop Trauma 20(4):253-60

19. Fankhauser F, Boldin C, Schippinger G, Haunschmid C, Szyszkowitz R (2005) A new locking plate for unstable fractures of the proximal humerus. Clin Orthop Relat Res 430:176-181

20. Kaukakis A, Apostolou CD, Taneja T, Korres DS, Amini A (2006) Fixation of proximal humerus fractures using the PHILLOS Plate. Early experience Clin Orthop 442:115-20

21. Edwards SL, Wilson NA, Zhang L, Flores S, Merk BR (2006) Two-part surgical neck fractures of the proximal part of the humerus. J Bone Joint Surg 88A:2258-64

22. Moda SK, Chadha NS, Sangwan SS, Khurana DK, Dahiya AS, Siwach RC (1990) Open reduction and fixation of proximal humeral fractures and fracture-dislocations. J Bone Joint Surg 72B:1050-2 